\title{
Calibration of antenna-radome and monument-multipath effect of GEONET_Part 2: Evaluation of the phase map by GEONET data
}

\author{
Yuki Hatanaka ${ }^{1}$, Masanori Sawada ${ }^{1}$, Akiko Horita ${ }^{1}$, Masaaki Kusaka ${ }^{1}$, James M. Johnson ${ }^{2}$, and Christian Rocken ${ }^{2}$ \\ ${ }^{1}$ Geographical Survey Institute, 1 Kitasato, Tsukuba, Ibaraki 305-0811, Japan \\ ${ }^{2}$ University Corporation for Atmospheric Research/GPS Science and Technology, 3300 Mitchell Lane, Boulder, CO 80301, U.S.A.
}

(Received May 15, 2000; Revised September 14, 2000; Accepted September 18, 2000)

\begin{abstract}
Phase maps for GSI/GEONET (Geographical Survey Institute/GPS Earth Observation NETwork) monuments and antennas obtained in Hatanaka et al. (2001) are evaluated by applying them to the analysis of GEONET. We used the same strategy as the GEONET routine analysis except for the antenna phase model. The coordinate solutions change by more than $10 \mathrm{~cm}$ in height when we apply the new phase maps. A scale change of up to $20 \mathrm{ppb}$ is also observed for one of the sub-networks. The height bias is not constant but changing daily and seasonably, which implies that seasonal variations in the GPS solution are related to mis-modeling of the phase characteristics and that other environmental or geometric factors are coupled to the phase mis-modeling. Two more checks are done by comparing tropospheric delay estimates, and by conducting elevation angle cutoff tests. Both tests show dramatic improvement when the new phase maps are applied, as compared to applying the standard antenna phase maps. It is concluded from this experiment with almost 1000 GEONET sites, that monument/antenna specific phase characteristic calibrations are essential for any application of GPS to achieve the highest accuracy for Earth science applications.
\end{abstract}

\section{Introduction}

In Part 1 companion of this paper (Hatanaka et al., 2001), we investigated the phase characteristics of GEONET monuments, and found that radome and signal scattering from the top of the stainless steel pillar severely affects the phase characteristics of the observable. The phase maps for typical monument types used within GEONET ("the new phase maps", hereafter) are obtained from the phase characteristics calibration experiments that were carried out using pillar models fabricated with the same material and having the same upper structure as the actual monuments. Although the new phase maps were validated with a short baseline in GSI, it is not clear whether the phase maps are applicable to the other GEONET stations. In this paper, we evaluate the impact of our new phase maps by applying them to the analysis of the whole GEONET network (Fig. 1) and comparing the results with the solutions obtained from using the old phase maps. In addition to examining the impact we also validate the new phase patterns by comparing solution statistics to those previously obtained with the old antenna patterns.

\section{Applying the New Phase Maps}

There are three main antenna types used in the GEONET network. It is well known that problems arise in double difference processing if the differences in antenna phase patterns are not accounted for (e.g. Meertens et al., 1996). This is because double differencing techniques rely on errors common to both sides of a baseline cancelling, which does not

Copy right(C) The Society of Geomagnetism and Earth, Planetary and Space Sciences (SGEPSS); The Seismological Society of Japan; The Volcanological Society of Japan; The Geodetic Society of Japan; The Japanese Society for Planetary Sciences. happen when different antenna types are combined. This so called antenna mixing problem can be overcome if accurate antenna phase maps are available (Mader, 1999). Since these were not available for each of the antenna types when GEONET began, the best available phase maps for the antennas from IGS_01 model (which was compiled by the International GPS Service: IGS) were used ("the old phase maps", hereafter) and to minimize the antenna mixing problem, the network was divided into three separate groups. Each of the sub-networks is processed independently holding a reference site in Tsukuba fixed. On the GSI campus in Tsukuba, one of each of the different antenna types is located within several hundred meters of the Tsukuba IGS site (TSKB). All these sites are tied together by L1 baselines without tropospheric parameter estimation, which greatly reduces errors due to antenna mixing. It was expected that the antenna mixing problem would be reduced to the mm-level caused only by the short L1 baselines that tied the three subnetworks together. However, we found that differences in the monumentation (radome used, diameter of the upper portion of the pillar, height of the antenna above the stainless steel base, etc.) within each of the three sub-networks had significant effects on the antenna phase patterns. This could be seen in baselines between sites having the same antenna type yet different monument construction. The monumentation within each of the three GEONET sub-networks was sufficiently different to affect the phase patterns of the antennas significantly. These findings are consistent with those of e.g. Elósegui et al. (1995) and Jaldehag et al. (1996).

To see the effect of the phase correction, the GEONET data are analyzed with old and new phase maps, respectively, and 


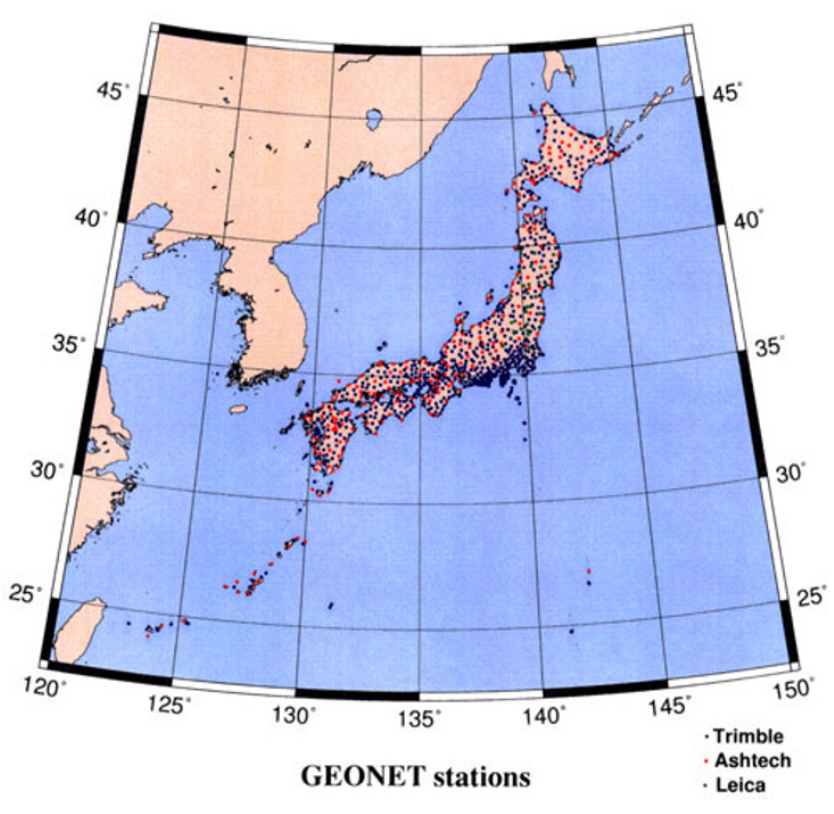

Fig. 1. Geographical distribution of GEONET stations. Three sub-networks that are classified by the antenna and receiver types: Trimble (blue), Ashtech (red), and Leica (green).
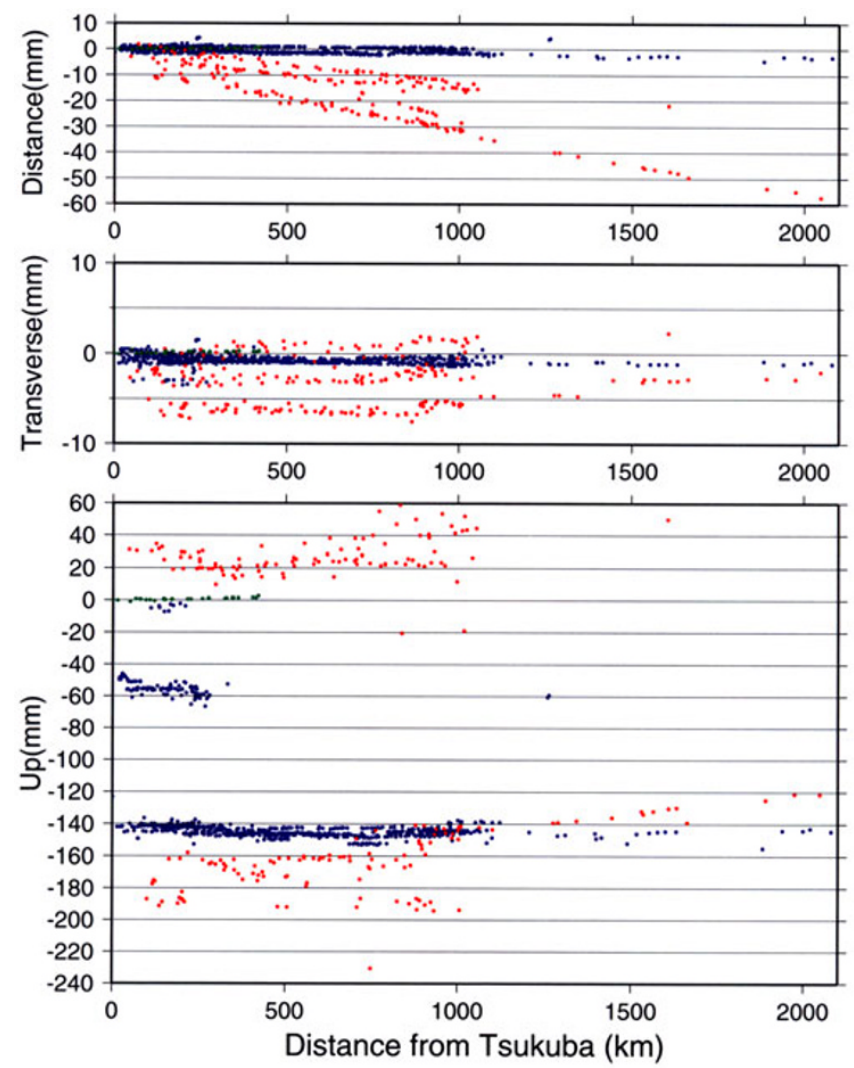

Fig. 2. Offset of coordinate estimates due to change of phase maps for Trimble sites (blue), Ashtech sites (red) and Leica sites (green). Elevation cutoff angle of 15 degrees is applied. Three components are shown: Horizontal components are decomposed into baseline length component from Tsukuba to each site (top) and transverse component (middle). The vertical component is shown at the bottom. the results are compared. The local tie data at Tsukuba was not changed even for the analysis with the new phase maps to isolate the effect of the network solution from that of the local tie. The error in local tie can cause small coordinate biases between the sub-networks. The bias due to the local tie will be discussed later in this paper.

Figure 2 shows the offsets of the coordinates due to the change of the phase map for all the GEONET stations. Large offsets up to $18 \mathrm{~cm}$ are shown in the height component. The amount of the offset differs by the antenna and monument types and is consistent with the results of the data analysis of the calibration experiment (Part 1, Hatanaka et al., 2001). Some Ashtech sites show a trend in the length component (distance of each station from Tsukuba), which implies that there is a scale bias of up to $30 \mathrm{ppb}(3 \mathrm{~cm}$ over $1000 \mathrm{~km})$ for this antenna/monument type.

\section{Phase Residuals}

If the new phase maps provide better corrections than the old ones, they should reduce post-fit phase residuals and improve carrier phase ambiguity resolution. To see the effect of new phase maps in the observable, the a posteriori rms of the single difference observable and the ratio of resolved ambiguities are averaged for each cluster for the old and new phase maps, respectively, and listed in Table 1 for one year. The average is taken within each network cluster. The results for the Leica cluster before March 17 was excluded from the statistics because of a data problem of these sites that happened sometimes during this period.

The GEONET network is processed by dividing the network into 9 clusters, the first containing Leica sites (C1), the next two Ashtech sites $(\mathrm{C} 2-\mathrm{C} 3)$ and the remaining are Trimble sites $(\mathrm{C} 4-\mathrm{C} 8, \mathrm{BB})$. The backbone cluster $(\mathrm{BB})$ contains three sites from each of the Trimble clusters $(\mathrm{C} 4-\mathrm{C} 8)$ and is used to tie the Trimble clusters together. The two Ashtech clusters are tied together by overlapping the two clusters. The average rms for the Ashtech clusters (C2 and C3) are reduced by $12-13 \%$.

The ratio of the number of resolved ambiguity is also slightly (1.0-2.1\%) improved for the Ashtech clusters. Examples of post-fit residuals for the baselines between old and newer sites of Ashtech from the analysis applying the new phase maps are shown in Fig. 3. Comparing them with the figure $2 \mathrm{~b}$ of Hatanaka et al. (2001), it is clear that the high amplitude low frequency error signals (which are indicative of either strong multipath or antenna mixing problems) are reduced by the new phase maps.

Although the improvement in the rms observable is not significant for any clusters other than $\mathrm{C} 2$ and $\mathrm{C} 3$, this does not mean that the effect of our new phase maps does not affect the estimation of coordinates and tropospheric delays. For these clusters, the un-modeled phase errors are absorbed by the adjusted parameters, and thus affect the estimated parameters. We will show how these parameters are improved by new the phase maps in the succeeding sections.

\section{Elevation Cutoff Angle Test}

One way to assess the validity of the new phase maps is to test the stability of the solution with respect to the satellite elevation cutoff angle. GEONET data from DOY 192, 1998 
Table 1. Comparison of results of the old phase maps and that of the new ones in terms of phase residuals and ratio of resolved ambiguities for one year (1998).

\begin{tabular}{|c|c|c|c|c|c|c|}
\hline \multirow[t]{2}{*}{ Cluster } & \multirow[t]{2}{*}{ Antenna } & \multirow[t]{2}{*}{ \# of sites } & \multicolumn{2}{|c|}{$\begin{array}{l}\text { rms phase } \\
\text { residuals }(\mathrm{mm})\end{array}$} & \multicolumn{2}{|c|}{$\begin{array}{l}\text { ratio of resolved } \\
\text { ambiguities }(\%)\end{array}$} \\
\hline & & & old PCV & new PCV & old PCV & new PCV \\
\hline $\mathrm{C} 1$ & LEIAT303 & 36 & 2.31 & 2.31 & 71.2 & 71.2 \\
\hline $\mathrm{C} 2$ & ASH700718A & 120 & 2.59 & 2.28 & 80.7 & 81.5 \\
\hline C3 & ASH700718A & 118 & 2.79 & 2.42 & 74.4 & 76.3 \\
\hline $\mathrm{C} 4$ & TRM23903.00 & 152 & 1.77 & 1.78 & 82.4 & 82.4 \\
\hline $\mathrm{C} 5$ & TRM23903.00 & 140 & 1.93 & 1.95 & 81.6 & 81.5 \\
\hline C6 & TRM23903.00 & 135 & 1.96 & 1.96 & 71.3 & 71.3 \\
\hline $\mathrm{C} 7$ & TRM23903.00 & 151 & 1.84 & 1.84 & 81.2 & 81.2 \\
\hline $\mathrm{C} 8$ & TRM23903.00 & 161 & 1.89 & 1.89 & 81.0 & 80.9 \\
\hline $\mathrm{BB}$ & TRM23903.00 & 20 & 2.02 & 2.00 & 57.9 & 57.9 \\
\hline
\end{tabular}
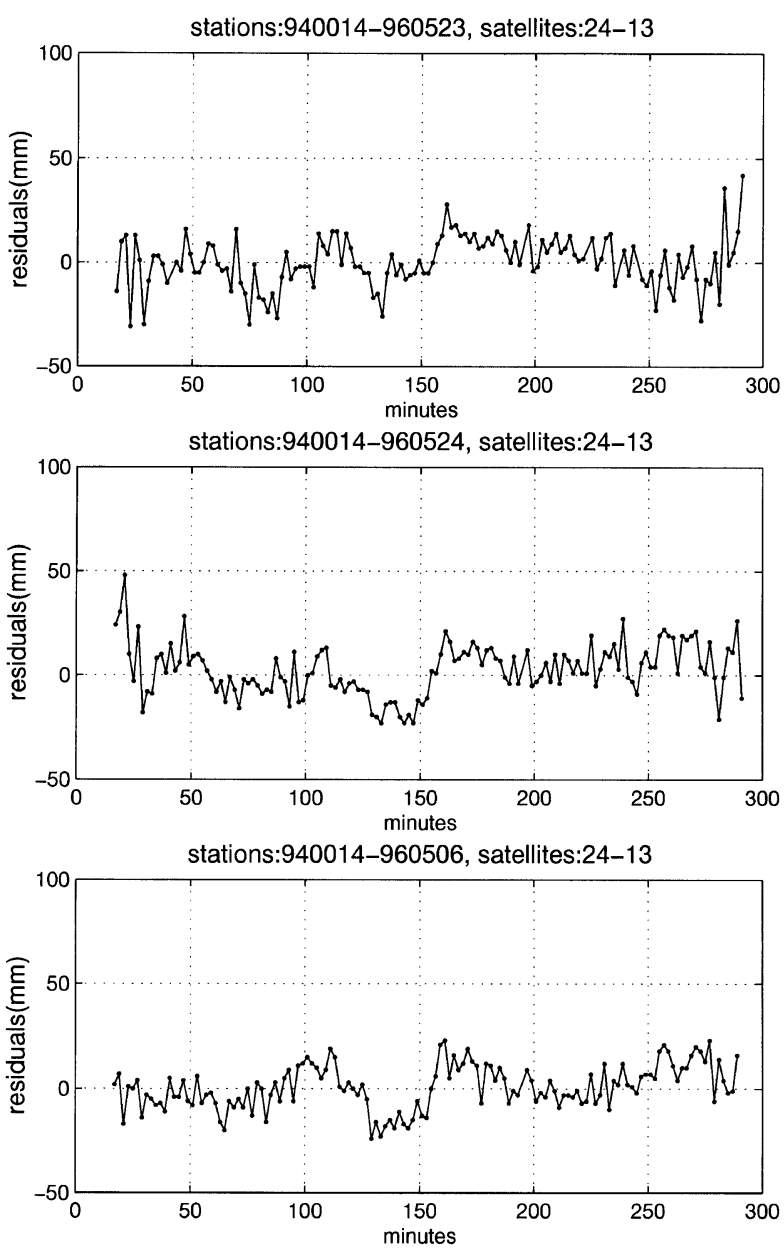

Fig. 3. Examples of post-fit residuals of double difference phase for the same data set as figure 2(b) of Hatanaka et al. (2001) from the analysis with the new phase maps applied.

are analyzed with elevation cutoff angles of 15, 20, and 25 degrees, respectively, applying both the old and new phase maps. The changes in coordinates with respect to the elevation cutoff using the old phase maps are plotted in Fig. 4 for all the sites. Here we take the solution with the cutoff angle of 15 degrees as a reference. With the old phase maps, the height coordinate changes with elevation cutoff angle by several $\mathrm{cm}$. A scale change of $20 \mathrm{ppb}$ is also seen for the Ashtech network in the figure. By applying the new phase maps (Fig. 5), the results are dramatically improved. For most of the sites, the difference in the height component is around $1-3 \mathrm{~cm}$ (22 $\mathrm{mm}$ of RMS) for the elevation cutoff angle of 20 degrees and no scale change is seen in the plot. There are several stations, which do not show improvement or become even worse. Some of the cases where there is no improvement can be explained by the fact that non-standard monuments for which we did not evaluate new phase maps were used for the site, and antenna height error is found for one of the other sites.

\section{Effect on Estimates of Tropospheric Delay}

Another test is to see if the troposphere delay estimate is reasonable or not. In general, un-modeled phase errors heavily affect the solutions when the tropospheric delay is estimated. Because of high correlation among the parameters, both vertical coordinates and tropospheric parameters are biased.

There are five GEONET sites on GSI's campus in Tsukuba. These sites are useful to assess the phase map effect on the tropospheric delay estimate. The difference of the delay among these sites should be negligible since their locations are within $300 \mathrm{~m}$. Figure 6 shows the time series of tropospheric delay estimated with the old and new phase maps. Discrepancies up to $5 \mathrm{~cm}$ among the sites are seen in the case of the old phase maps, while the parameters are more consistent within $2 \mathrm{~cm}$ when the new phase maps are applied. The deviation of each plot from the average of the 4 sites is shown in Fig. 7. Since the scatter of 96TKB4 (Leica site) is relatively high, possibly due to the small network size $(\sim 450$ $\mathrm{km}$ ) of the Leica sub-network, this site is excluded from the calculation of the average to avoid the reference value to be affected by the noisy data. The discrepancy in the bias among the sites is $46 \mathrm{~mm}$ in maximum for the old phase maps and is reduced to $12 \mathrm{~mm}$ by applying the new phase maps. The 


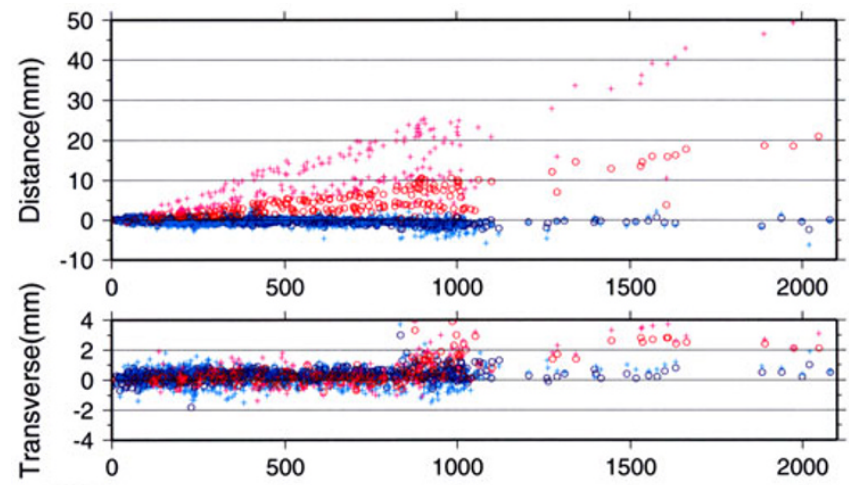

Total Zenith Delay at 5 Sites in Tsukuba

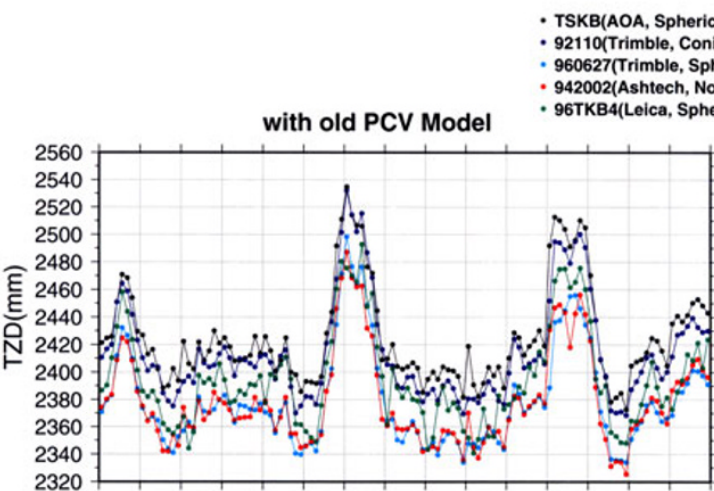

$\begin{array}{llllllllllllllll}80 & 81 & 82 & 83 & 84 & 85 & 86 & 87 & 88 & 89 & 90 & 91 & 92 & 93 & 94 & 9\end{array}$

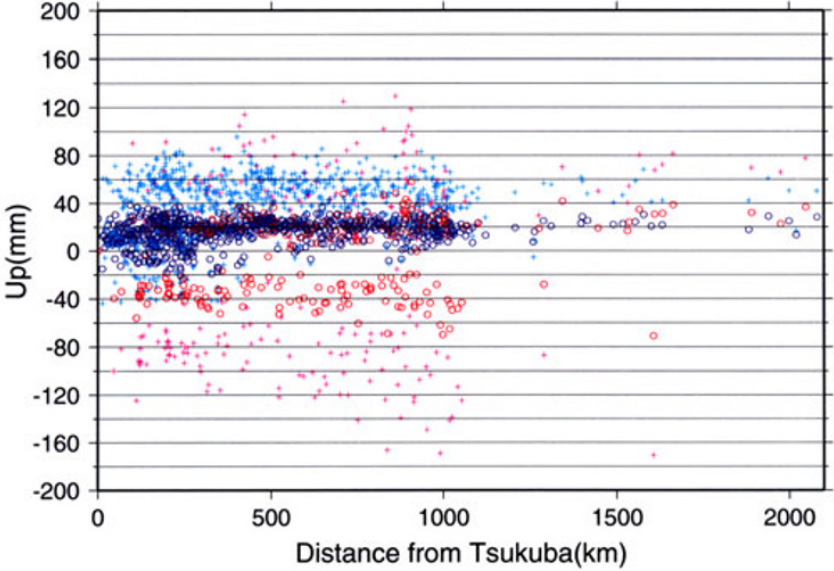

with new PCV Model

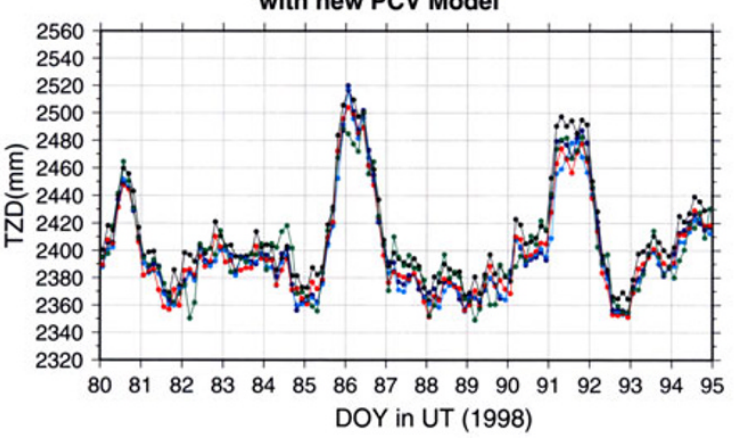

Fig. 4. Change in coordinate estimates by changing the elevation cutoff angle from 15 degrees to 20 degrees (circles) or 25 degrees (cross) for the old phase maps. The red and pink symbols are for Ashtech sites and blue and magenta symbols for other sites (lumped together). The definition of the three components is the same as in Fig. 2.

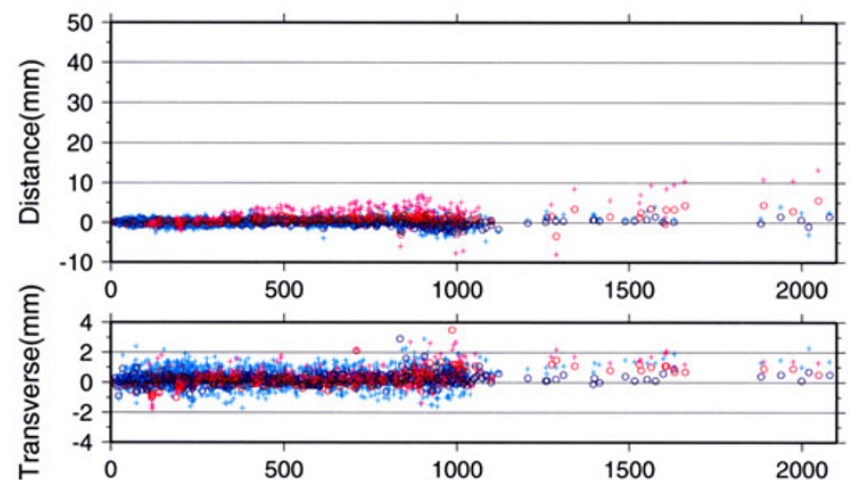

Deviation of Total Zenith Delay at 5 Sites in Tsukuba Reference: averaged time-series (96TKB4 is excepted)

ig. 6. Troposphere delay estimated at 5 nearby sites in GSI at Tsukuba, Japan, with the old phase maps (top) and that with the new phase maps (bottom).
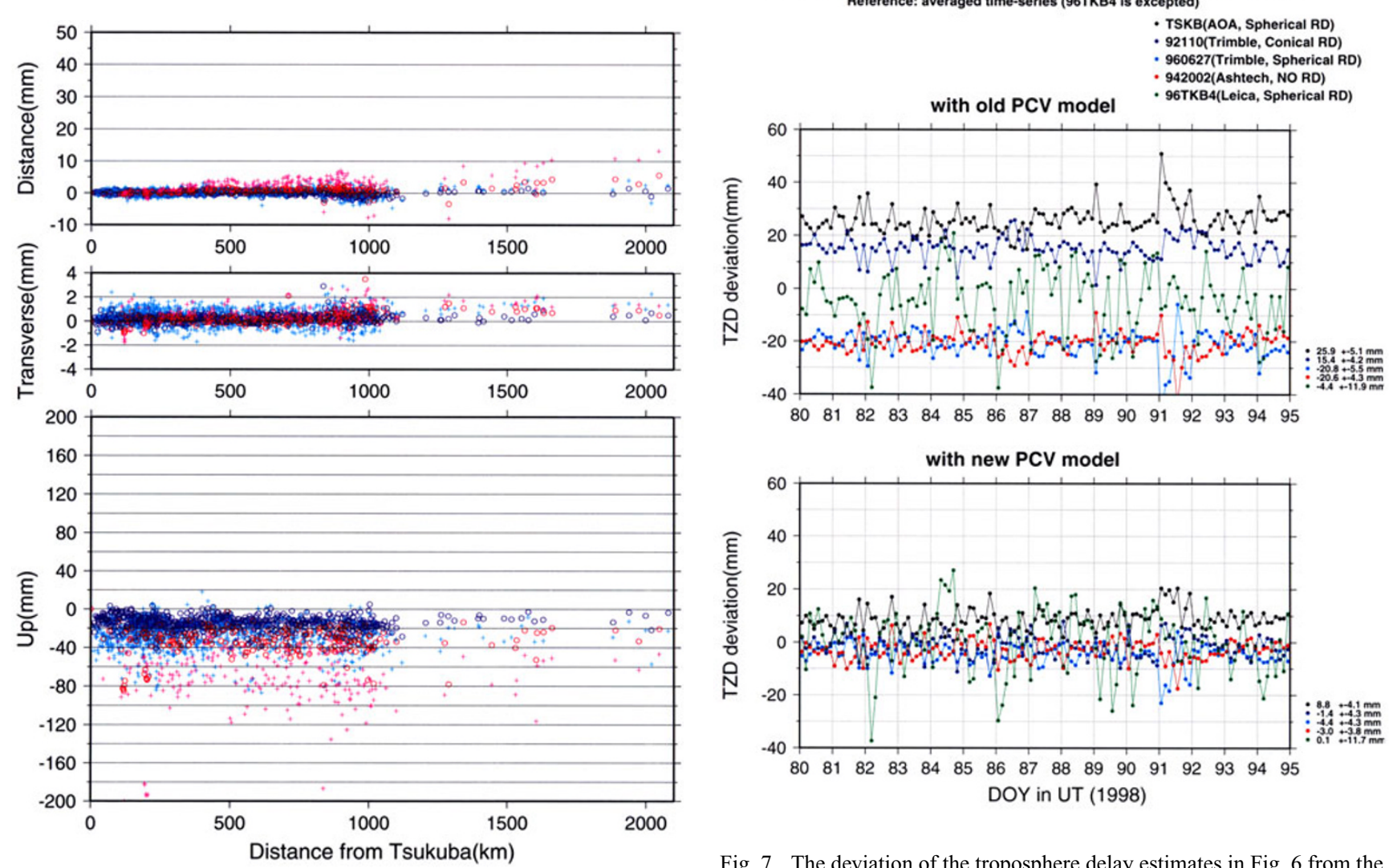

Fig. 7. The deviation of the troposphere delay estimates in Fig. 6 from the average. The biases and the standard deviations are shown at the right of the graphs. 

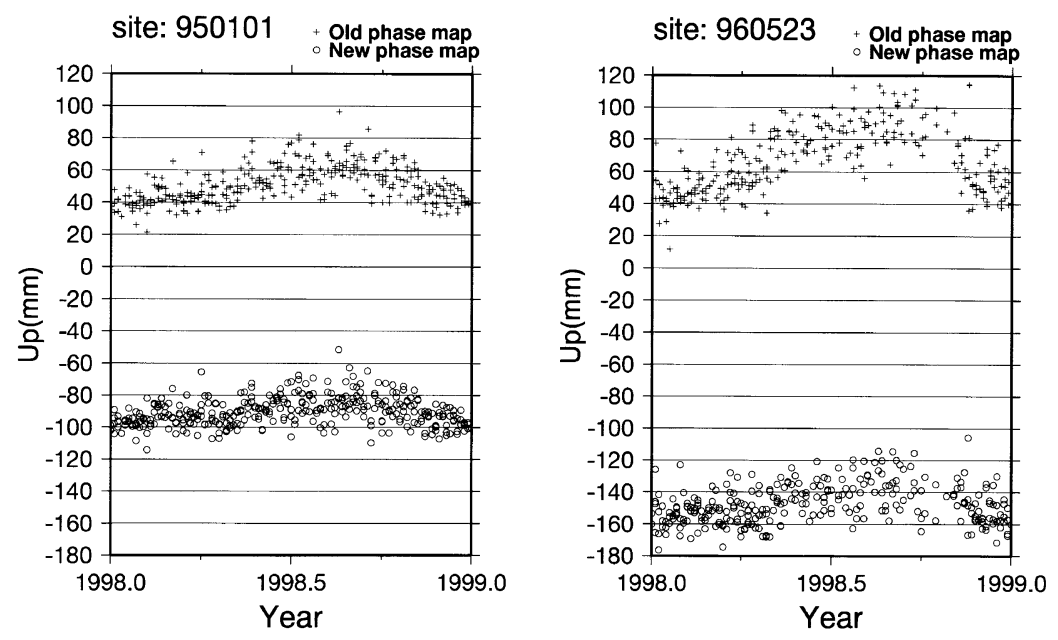

Fig. 8. Comparison of the time series of vertical coordinate solved by the old phase models (cross) and that by the new models (open circle) for the site 950101 (Trimble, monument type: 95) and that for the site 960523 (Ashtech, monument type: 95).
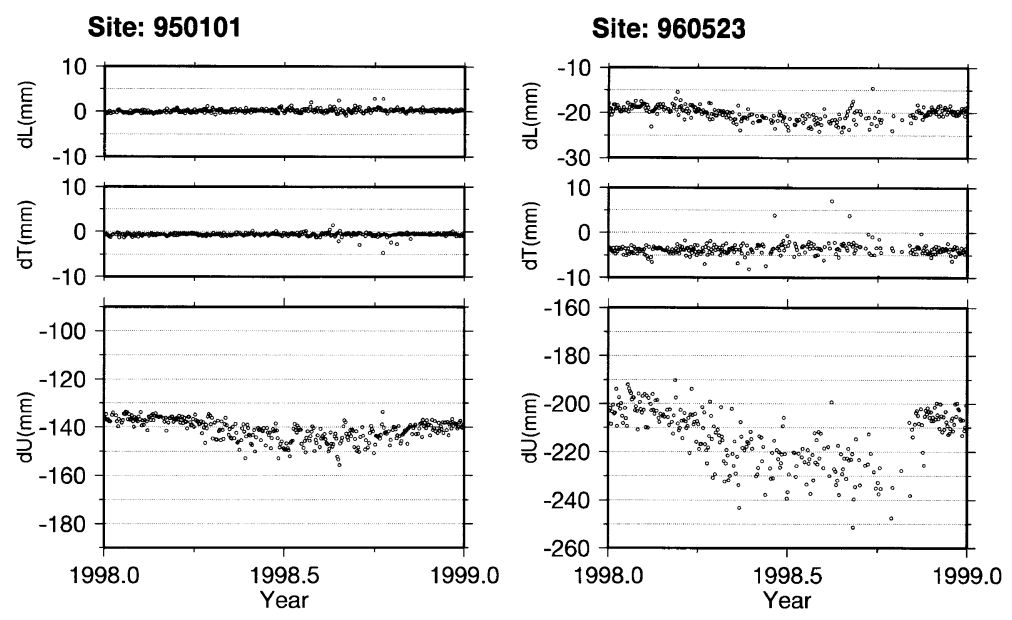

Fig. 9. Time series of coordinate bias due to the difference of the phase maps for the same sites as in Fig. 8.

standard deviations of the biases are also slightly reduced. This indicates that we have greatly improved the consistency of the estimate of the tropospheric delay and marginally improved the random noise component of the estimates.

\section{Long-Term Stability of the Coordinate Bias}

It is important to check if the biases in the parameters due to un-modeled phase errors remain constant for long multiyear periods. If they are constant, then we can apply constant offsets to correct the old solutions. The constant bias can also be neglected in the case of monitoring of crustal deformation for which only the change of baseline components is of importance.

One year (1998) of GEONET were analyzed using old and new phase maps, respectively. Examples of time series of the height component with both phase maps are compared in Fig. 8. The antenna types of the sites are Trimble Permanent L1/L2 (TRM2399903.00) for the site 950101 and Ashtech 700718A (ASH700718A) for the site 960523 and the monuments of the both sites are type- 95 with hemisphere radomes
(\#3 and \#5 in the table 1 of Hatanaka et al., 2001). Large biases between the two phase models are obvious for both sites, which is also seen in Fig. 2. Moreover, the amplitude of the annual variation that appears in the plots are reduced by applying new phase maps. By taking the difference between the two cases, a one-year time series of the coordinate biases are obtained for each site (Fig. 9). The biases in horizontal components are also shown in Fig. 9. Annual variation with the amplitude of 1-2 cm is clearly seen in the height biases. The horizontal scale bias for the site 960523 also contains an annual term with the amplitude of a few $\mathrm{mm}$. Since the only difference in the analysis are the phase map models and since the models do not vary with time (the mapping function used here doesn't contain any temporal variation), it must be assumed that errors in the phase maps are correlated with other error sources. The results imply that the mis-modeling of the phase characteristics is not solely the cause of this variation, but other environmental or geometric factors must be coupled to it. The fact that the biases show higher scatter during the summer season when the humidity and its variability is high, 
suggests that the troposphere may be a contributing factor.

The seasonal variations of the coordinates are seen in many GPS network analysis results and its cause is not yet well understood. The above results imply that the un-modeled antenna phase map error is partly responsible for it. Whatever the true causes are, the precise correction for the phase characteristics is mandatory for further investigation on the seasonal variation of the GPS solutions.

\section{Repeatability of Coordinates}

The temporal change of the coordinate biases (Fig. 9), especially of the height components, contains not only the seasonal variation but also short-term scattering. In this section, we evaluate the effect of the new phase maps in terms of repeatability of coordinates.

To exclude the annual signals and trends from the evaluation of the repeatability, the standard deviations are calculated as follows. First, monthly values of coordinate variance are calculated for each site and from the solutions obtained by using old and new phase maps, respectively. Then, the monthly variance is stacked for one year with appropriate re-normalization, and standard deviation (S.D.) is obtained as the square root of it by following equation.

$$
\text { S.D. }=\sqrt{\frac{1}{N-M} \sum_{m=1}^{M}\left(n_{m}-1\right) \cdot \sigma_{m}^{2}}
$$

where $\sigma_{m}^{2}$ and $n_{m}$ are variance of the coordinates and number of days, respectively, in the $m$-th month of the year. $N$ is summation of $n_{m}$ for $M$ months, i.e. number of days in the year. $M$ is the number of months in which usable coordinates are available (usually 12).

The averaged S.D. values over all sites are listed in Table 2 . The reduction of the S.D. values is very little for horizontal components, but S.D. value is reduced by $0.64 \mathrm{~mm}(6.1 \%)$ for the vertical component. The histogram of the S.D. reduction is shown in Fig. 10. The improvement is clear for the vertical component by the shift of the peak of the histogram while the peaks for the horizontal components do not shift. Although the improvement is small, it demonstrates the new phase maps provides statistically better repeatability of vertical coordinates than the old ones. It is no wonder that the effect of the new phase maps is little on the horizontal component since the effect to the horizontal component is likely to be averaged out by the fact that azimuthal asymmetry of the phase characteristics is not taken into account.

The above results together with the annual component in the height bias imply that the antenna-monument phase corrections are important for geodetic, crustal deformation, and meteorological applications of GEONET.

Table 2. Mean S.D. of coordinates in millimeters

\begin{tabular}{lccc}
\hline & NS & EW & UD \\
\hline Old phase maps & 2.31 & 2.10 & 10.41 \\
New phase maps & 2.30 & 2.09 & 9.78 \\
\hline difference & 0.01 & 0.01 & 0.64 \\
\hline
\end{tabular}
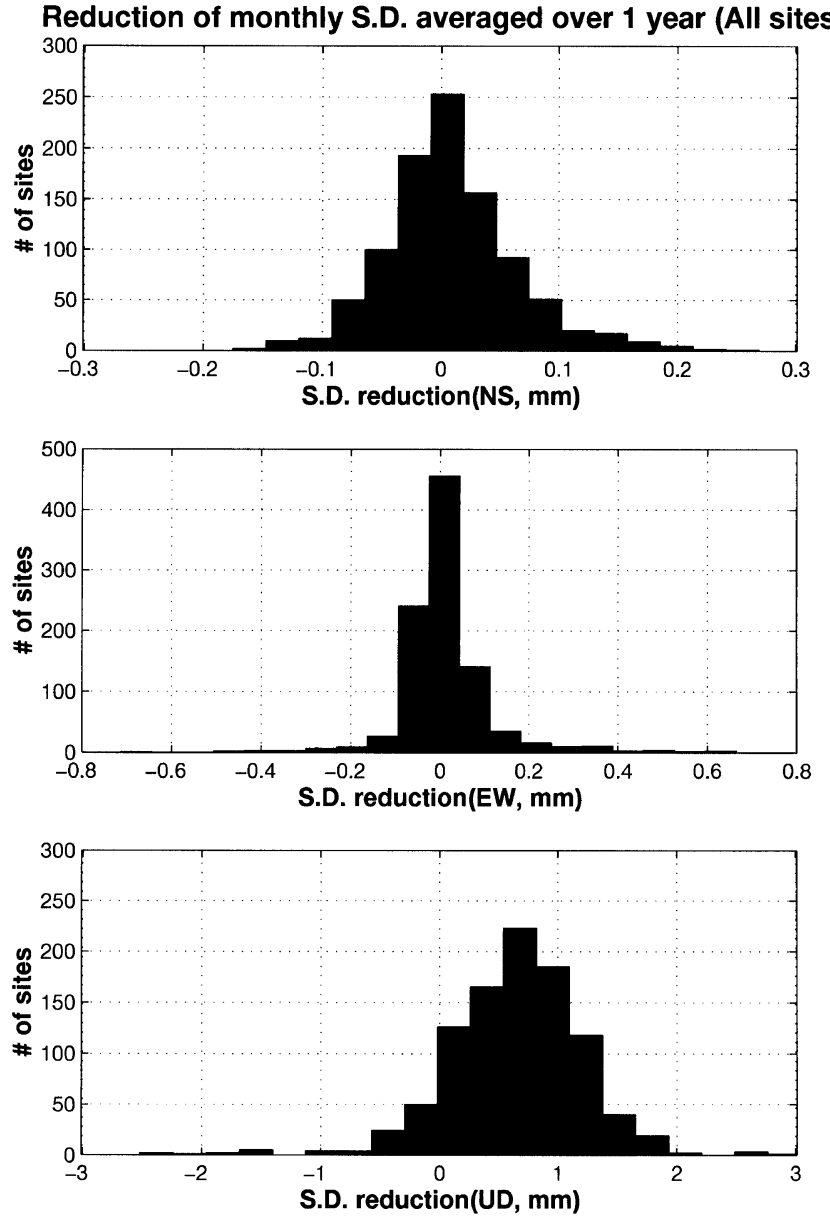

Fig. 10. Histograms of S.D. reduction of site coordinates by applying new phase maps for north-south component (top), for east-west component (middle) and up-down component (bottom).

\section{Discussion}

The new phase maps are specific for the type of the GEONET site (one for each monument/antenna type combination). In reality, since the site environment is different from place to place, the phase characteristics can be different for individual sites with identical monument/antenna types. The results of elevation cutoff tests, however, show that the new phase maps improve the consistency of the coordinates to a few $\mathrm{cm}$ with only a few exceptions. This fact implies that the phase characteristics of GEONET sites with the same monument-antenna type are relatively homogeneous within this level.

As shown in Fig. 2, neglecting the dependence of phase characteristics on the monument types results in the large coordinate bias, especially, in the height component. This height bias is not only systematic but also dependent on monument types. It is particularly important to eliminate this error for geoid modeling and for defining a vertical reference frame. GEONET is regarded as a powerful tool to realize a geometric reference frame of Japan with high spatial density. Relative geoid heights can be estimated by differencing the leveled height and ellipsoidal (GPS) height at the same markers (e.g. Hofmann-Wellenhof et al., 1997). This method is independent of using satellite or surface gravity data to es- 
Table 3. Bias in local tie due to change in phase maps from old ones to new ones.

\begin{tabular}{|c|c|c|c|c|c|c|c|}
\hline \multicolumn{2}{|c|}{ Baseline } & \multicolumn{6}{|c|}{ bias (mm) } \\
\hline from & to & $\mathrm{X}$ & $\mathrm{Y}$ & Z & North & East & Up \\
\hline TSKB & 92110 & 2.9 & -3.1 & -2.8 & 0.20 & 0.52 & -5.05 \\
\hline TSKB & 942002 & 1.2 & -1.1 & 0.2 & 1.12 & 0.07 & -1.20 \\
\hline TSKB & 96TKB4 & -20.7 & 20.1 & 20.0 & -0.69 & -2.14 & 35.04 \\
\hline
\end{tabular}

timate a geoid model, and is often used for evaluating the quality of geoid models derived from gravity data. However, it becomes evident from this study that a site-specific height bias of up to $20 \mathrm{~cm}$ can be introduced into the ellipsoidal height if GEONET data are used without correcting for the effect of the monument-dependent phase error. This level of inconsistency cannot be neglected since the comparison of geoid undulation between both technique is made nowadays in $10 \mathrm{~cm}$ level in Japan after removing tilting component (Kuroishi, 1995, 2000) or in 20-30 cm level over continental scales in well resolved areas after removing datum offsets (e.g. Milbert, 1995; Lemoime et al., 1997). The correction of phase characteristics is, thus, mandatory for the evaluation of geoid models with the GPS/leveling technique and for future application of geoid calculations to integrate GPS/leveling data for defining a vertical datum.

The official IGS model for the Dorne Margolin T antenna is applied for the phase characteristics of the reference antenna to obtain the new phase maps (Hatanaka et al., 2001). Although this model assumes nominal phase center offsets and no phase center variation for this antenna type, this is known to be wrong. In fact, the absolute phase patterns of Dorne Margolin T antennas measured in anechoic chambers from several calibration experiments shows the phase center variation over a few cm level (Clark and Schupler, 1996; Meertens et al., 1996; Rocken et al., 1995). The errors in absolute antenna phase patterns are not a problem for short baselines because they are canceled out by double difference operation. It can be a problem for long baselines however, because the elevation angle of a satellite is not the same at both ends of long baselines and elevation dependent effects do not cancel. Rothacher et al. (1995) tested this issue by analyzing a $1200 \mathrm{~km}$ baseline with changing the absolute pattern of the antenna phase model. Their results show a bias of about 1.5 $\mathrm{cm}$ (or $0.012 \mathrm{ppm}$ ) in the baseline length. However, since the GPS satellite orbit information and the earth rotation parameters are also analyzed by assuming the IGS_01 model by IGS analysis centers, it is reasonable choice to use the same model for the reference antenna to maintain consistency with the IGS products. The issue of absolute calibration of GPS antennas is important not only for the analysis of GEONET but also for the analysis of the global GPS network by the IGS, and further study is needed to assess the impact of this issue and to find a better solution. Once reliable absolute phase maps are available for the reference antenna in future, it will be possible to apply them to the GEONET processing.

Finally, we again notice that, in this paper, the local tie data to combine the three sub-networks are not corrected for the phase maps. For completeness, the biases in the local tie vectors due to changing phase maps from the old ones to the new ones are calculated. The baselines from the Tsukuba IGS site (TSKB) to the anchor sites of the three sub-networks are analyzed by using two year data from 1998 to 1999 with single frequency (L1) without estimating tropospheric delay by applying old and new phase maps, respectively. The coordinate biases between two cases are listed in Table 3 .

\section{Conclusions}

We have tested the new phase maps by applying it to the GEONET data. The fit to the phase observables is improved, more ambiguities are resolved, the coordinates become more independent of the elevation cutoff angle, repeatability of vertical component is improved, and discrepancies in troposphere delay estimates are reduced. The results clearly show the superiority of the new phase maps in both the observable domain and the model parameter domain.

Since the carrier phase is the primary observable of GPS, mis-modeling of it affects a wide area of the applications of GPS. The results obtained here have a large impact on many applications of GEONET such as constructing a regional reference frame, GPS meteorology, collocation with other space geodetic techniques, geoid leveling, and so on.

Acknowledgments. The first author would thank to Dr. Kuroishi of Geographical Survey Institute for discussion on the impact of the results on the geoid studies. This study was partly supported by Science and Technology Agency of Japanese Government under the Special Coordination Funds for the Science on GPS meteorology project.

\section{References}

Clark, T. A. and B. R. Schupler, What Are Phase-Center Variations and Why Should I Worry?, Proceedings of IGS analysis Center Workshop, Silver Springs, MD, U.S.A, June 1996, International GPS Service, pp. 107-118, 1996.

Elósegui, P., J. L. Davis, R. T. K. Jaldehag, J. M. Johansson, A. E. Niell, and I. I. Shapiro, Geodesy using the Global Positioning System: The effect of signal scattering on estimates of site position, J. Geophys. Res., 100, 9921-9934, 1995.

Hatanaka, Y., M. Sawada, A. Horita, and M. Kusaka, Calibration of antennaradome and monument-multipath effect of GEONET-Part 1: Measurement of phase characteristics, Earth Planets Space, 53, this issue, 13-21, 2001 .

Hofmann-Wellenhof, B., H. Lichtenegger, and J. Collins, GPS Theory and Practice, 4th edition, pp. 389, Springer Wien New York, 1997.

Jaldehag, R. T. K., J. M. Johansson, B. O. Ronnang, P. Elosegui, J. L. Davis, I. I. Shapiro, and A. E. Niell, Geodesy using the Swedish permanent GPS network: effect of signal scattering on estimates of relative site positions, J. Geophys. Res., 101, 17841-17860, 1996.

Kuroishi, Y., Precise Gravimetric Determination of Geoid in the Vicinity of Japan, Bull. Geographical Survey Inst., 41, 1-93, 1995.

Kuroishi, Y., Gravity Field and Geoid for Japan, in International Association of Geodesy Symposia, Vol. 121, edited by Schwartz, pp. 149-154 Geodesy Beond 2000 - The Challenges of the First Decade, SpringerVerlag, Berlin Heidelberg, 2000 
Lemoine, F. G., D. E. Smith, L. Kunz, R. Smith, E. C. Pavlis, N. K. Pavlis, S. M. Klosko, D. S. Chinn, M. H. Torrence, R. G. Williamson, C. M. Cox, K. E. Rachlin, Y. M. Wang, S. C. Kenyon, R. Salman, R. Trimmer, R. H. Rapp, and R. S. Nere, The Development of the NASA GSFC and NIMA Joint Geopotential Model, in International Association of Geodesy Symposia, Vol. 117, edited by J. Segawa, H. Fujimoto, and S. Okubo, Gravity, Geoid and Marine Geodesy (1996 Tokyo), SpringerVerlag, Berlin Heidelberg, 1997.

Mader, G., GPS Antenna Calibration at National Geodetic Survey, GPS Solutions, 3, 50-58, 1999.

Meertens, C., C. Alber, J. Brown, C. Rocken, B. Stephens, R. Ware, M. Exner, and P. Kolesnikoff, Field and Anechoic Chamber test, Proceedings of IGS analysis Center Workshop, Silver Springs, MD, U.S.A, June 1996 , International GPS Service, pp. 107-118, 1996.

Milbert, D., Improvement of a high resolution geoid height model in the United States by GPS height on NAVD88 benchmarks, IGeS Bulletin,
N. 4, "New Geoid in the World", 13-36, 1995.

Rocken, C., C. Meertens, B. Stephens, J. Braun, T. Van Hove, S. Perry, O. Ruud, M. MacCallum, and J. Richardson, UNAVCO Academic Research Infrastructure (ARI) Receiver and Antenna Test Report, University Corporation for Atmospheric Research/University NAVSTAR Consortium (internal document), 1995.

Rothacher, M., S. Schaer, L. Mervart, and G. Beutler, Determination of Antenna Phase Center Variations Using GPS data, in IGS Workshop Proceedings on Special Topics and New Directions, edited by G. Gendt and G. Dick, pp. 77-92, GeoForschungsZentrum, Potsdam, Germany, May 15-18 1995, 1995.

Y.Hatanaka (e-mail: hata@gsi.go.jp), M. Sawada, A. Horita, M. Kusaka, J. M. Johnson, and C. Rocken 